\title{
Medizin am Wendepunkt
}

\section{Adrian Ritter}

Freier Journalist

\section{Stellen in Zukunft Algorithmen statt Ärzte Diagnosen? Werden digitale Daten das medizinische Personal administrativ entlasten? Eine Tagung des «Health Ethics and Policy Lab» der Universität Zürich reflektierte die Chancen und Herausforderungen der Digitalisierung im Gesundheitswesen.}

Wird es ein Befreiungsschlag? Die Ärztinnen und Ärzte dreier Londoner Spitälern sollen in Zukunft mehr als 500000 Arbeitsstunden pro Jahr für den Kontakt mit den Patienten statt für administrative Arbeiten nutzen können. Möglich machen soll dies die Zusammenarbeit des National Health Service (NSH) mit «DeepMind», der Google-Firma für künstliche Intelligenz. DeepMind erhält ab 2017 die Gesundheitsdaten von 1,6 Millionen Patienten der beteiligten Spitäler - von der Krankengeschichte bis zu aktuellen Blutwerten. Die App «Stream» wird die Daten auf Anzeichen eines akuten Nierenversagens oder einer Blutvergiftung analysieren und Ärzte und Patienten frühzeitig warnen. Bis zu 10000 Todesfälle pro Jahr können so verhindert werden, schätzt Deepmind. Als willkommener Nebeneffekt soll dank der digitalen Datenflüsse der administrative Aufwand kleiner werden. Das Beispiel zeigt: Die Digitalisierung des Gesundheitswesens ist in vollem Gange. Das wurde auch Anfang Dezember an der Tagung «Digital Health: Exploring Ethics and Policy» an der Universität Zürich klar.
Marcel Salathé, Leiter des Digital Epidemiology Lab an der ETH Lausanne, berichtete dabei von einer Software, die mit 99-prozentiger Trefferquote Pflanzenkrankheiten aufgrund von Fotos der Pflanzenblätter erkennen kann. Was bei Pflanzen funktioniert, wird auch beim Menschen erprobt: Salathé zitierte eine Studie, gemäss der die Erfolgsquote eines Algorithmus beim Erkennen von Hautkrebs zwar nur bei 60 Prozent

\section{Die Digitalisierung des Gesundheitswesens ist} in vollem Gange.

lag - Dermatologen allerdings schnitten mit 46 Prozent noch schlechter ab. Je mehr Bilder die Software gefüttert bekommt, desto besser werden die Ergebnisse «deep learning» nennen das die Datenspezialisten. «In den kommenden Jahren werden Tausende von Studien zeigen, dass Algorithmen gewisse Aufgaben besser erledigen können als der Mensch», ist Marcel Salathé überzeugt. Dabei ist für ihn allerdings klar, dass Algorithmen nicht eine definitive Diagnose stellen,

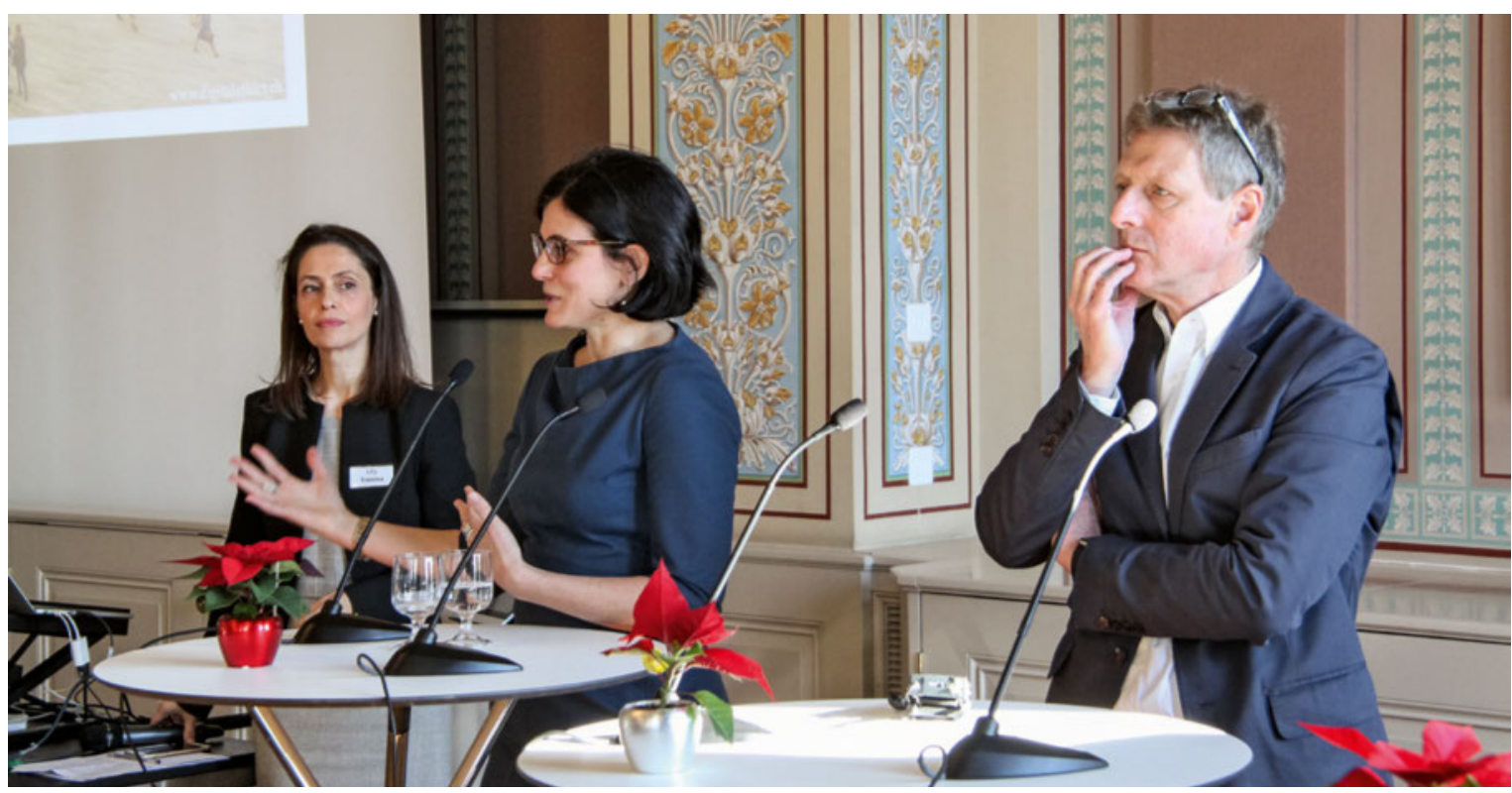

Abbildung 1: Von links: Tagungsorganisatorin Effy Vayena (UZH), Tania Simoncelli (MIT) und Ernst Hafen (ETH Zürich). (Bild: Adrian Ritter) 
sondern Hinweise geben sollen, wann beispielsweise eine Gewebeprobe bei Krebsverdacht sinnvoll sei.

Big Data erobert das Gesundheitswesen - nicht nur bei der Auswertung von bildgebenden Techniken, sondern auch in der Form von Apps auf dem Smartphone. Die Vermessung des eigenen Körpers ist in vollem Gange und wird von Krankenkassen bisweilen finanziell belohnt. Die CSS gewährt Zusatzversicherten einen Prämienrabatt von 40 Rappen für jeden Tag, an dem eine App 10000 Schritte zählt. Firmen forschen zudem daran, wie Smartphone-Besitzer in Zukunft sogar ein EKG zuhause erstellen können.

\section{Krankheiten verstehen}

Effy Vayena ist Ethikerin und leitet das neu gegründete «Health Ethics and Policy Lab» der Universität Zürich, das die Tagung organisiert hatte. Sie ist überzeugt: "Medizin und Gesundheitsversorgung befinden sich an einem Wendepunkt: die Digitalisierung ermöglicht ein bisher unerreichtes Verständnis der Ursachen von Krankheiten und erlaubt es, neue Behandlungsmöglichkeiten zu entwickeln. Gleichzeitig stellt die Digita-

Big Data erobert das Gesundheitswesen - nicht nur bei der Auswertung von bildgebenden Techniken, sondern auch in der Form von Apps auf dem Smartphone.

lisierung unsere Gesellschaft vor grosse Herausforderungen ethischer und rechtlicher Art.»

Das betrifft etwa den Schutz der Privatsphäre, aber auch den Schutz der Daten selber. Gemäss dem Cyber Security Intelligence Index 2016 von IBM ist die Gesundheitsbranche inzwischen das weltweit attraktivste Angriffsziel für Cyberkriminelle.

\section{Die Schweiz im Hintertreffen}

Die Schweiz sei nicht vorne mit dabei bei der Digitalisierung im Gesundheitswesen, meinte Antoine Geissbühler, Professor am Departement Radiologie und medizinische Informatik an der Universität Genf. Für Peter Meier-Abt, Vizepräsident der Schweizerischen Akademie der Medizinischen Wissenschaft, sind die Universitäten und Spitäler in der Schweiz zu wenig vernetzt und nutzen eine zu grosse Vielfalt an inkompatiblen Systemen der Datenverwaltung. Abhilfe soll die Nationale Förderinitiative "Personalisierte Medizin" und das daraus entstandene «Swiss Personalized Health Network» schaffen. In einem nächsten Schritt gelte es dann, die Gesundheitsdaten international zu vernetzen. Einen bedeutenden Schritt der Digitalisierung

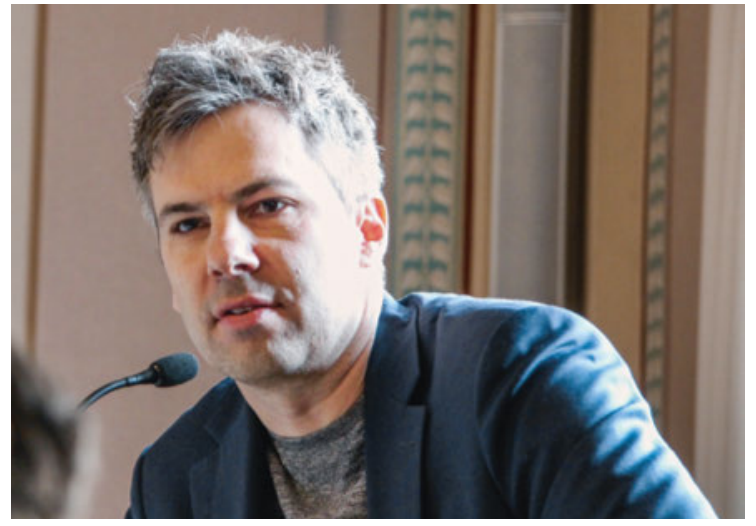

Abbildung 2: Marcel Salathé (Bild: Adrian Ritter)

habe die Schweiz mit der beschlossenen Einführung des elektronischen Patientendossiers gemacht, sagte Google-Vertreter Daniel Schönberger an der Tagung. Das entsprechende Gesetz soll 2017 in Kraft treten. In den USA sei die Digitalisierung im Gesundheitswesen schneller vonstatten gegangen, berichtete Tania Simoncelli vom Massachusetts Institute of Technology (MIT). Seit dem Amtsantritt von Barack Obama habe die US-Regierung mehr als 29 Milliarden Dollar in die Digitalisierung des Gesundheitswesens investiert. Gewisse Technologiefirmen, Spitäler und Labors blockieren den Datenaustausch allerdings, weil sie um ihr Business fürchten, wenn sie Daten mit Konkurrenten teilen. «Die Schweiz tut gut daran, die ganzen Fragen im Zusammenhang mit der Digitalisierung des Gesundheitswesen zwar langsamer, aber dafür sinnvoller zu regeln", gab Simoncelli zu Bedenken.

\section{Genossenschaftliche Lösung}

Ansätze einer sicheren, gesellschaftlich sinnvollen Regelung der digitalen Gesundheitsdaten seien in Sicht, wurde an der Tagung von verschiedenen Referierenden betont. Ein positives Beispiel sei die Initiative Midata.coop von Ernst Hafen, Professor am Institut für Molekulare Systembiologie der ETH Zürich. Midata ist ein genossenschaftliches Projekt, bei dem die Bürgerinnen und Bürger ähnlich einem Bankkonto ihre Daten sicher und zentral hinterlegen und selbst bestimmen könnten, wer Zugang dazu erhalten soll. Allfällige kommerzielle Erträge aus der Datennutzung werden in Forschungsobjekte reinvestiert, die der Allgemeinheit zugute kommen.

Ob Ärztinnen und Ärzte dank der Digitalisierung in Zukunft aber tatsächlich mehr Zeit für ihre Patienten haben werden, steht noch in den Sternen - beziehungsweise wird eher in der politischen Sphäre denn in den Technologie-Labors entschieden. 